TRANSACTIONS OF THE

AMERICAN MATHEMATICAL SOCIETY

Volume 363, Number 3, March 2011, Pages 1665-1686

S 0002-9947(2010)05244-4

Article electronically published on October 15, 2010

\title{
REFINEMENTS OF THE LITTLEWOOD-RICHARDSON RULE
}

\author{
J. HAGLUND, K. LUOTO, S. MASON, AND S. VAN WILLIGENBURG
}

\begin{abstract}
In the prequel to this paper, we showed how results of Mason involving a new combinatorial formula for polynomials that are now known as Demazure atoms (characters of quotients of Demazure modules, called standard bases by Lascoux and Schützenberger) could be used to define a new basis for the ring of quasisymmetric functions we call "Quasisymmetric Schur functions" (QS functions for short). In this paper we develop the combinatorics of these polynomials further, by showing that the product of a Schur function and a Demazure atom has a positive expansion in terms of Demazure atoms. We use these techniques, together with the fact that both a QS function and a Demazure character have explicit expressions as a positive sum of atoms, to obtain the expansion of a product of a Schur function with a QS function (Demazure character) as a positive sum of QS functions (Demazure characters). Our formula for the coefficients in the expansion of a product of a Demazure character and a Schur function into Demazure characters is similar to known results and includes in particular the famous Littlewood-Richardson rule for the expansion of a product of Schur functions in terms of the Schur basis.
\end{abstract}

\section{INTRODUCTION}

A composition (weak composition) with $n$ parts is a sequence of $n$ positive (nonnegative) integers, respectively. A partition is a composition whose parts are monotone non-increasing. If $\tau$ is a weak composition, composition, or partition, we let $\ell(\tau)$ denote the number of parts of $\tau$. Throughout this article $\gamma$ is a weak composition with $\ell(\gamma)=n$, while $\beta$ and $\lambda$ denote compositions and partitions, respectively, with $\ell(\beta) \leq n, \ell(\lambda) \leq n$. The polynomials in this paper (Schur functions, Demazure atoms and characters, QS functions) depend on a finite set of variables $X_{n}=\left\{x_{1}, x_{2}, \ldots, x_{n}\right\}$ which we often omit for the sake of readability.

Symmetric functions in a set of variables $X_{n}$ play a central role in representation theory, and in recent years have found increasing utility in several other branches of mathematics and physics such as special functions, algebraic geometry, and statistical mechanics. One of the most general symmetric functions is a family of orthogonal polynomials $J_{\mu}\left(X_{n} ; q, t\right)$ introduced by Macdonald [12, [13] in 1988, which depend not only on $X_{n}$ but also on a partition $\mu$ and two extra parameters $q, t$. The $J_{\mu}$ contain many of the most useful symmetric functions such as limiting or special cases. In 1995 Macdonald [14 introduced a very general family of orthogonal polynomials called the non-symmetric Macdonald polynomials $E_{\gamma}\left(X_{n} ; q, t\right)$ which, although not symmetric functions, satisfy versions of most

Received by the editors July 10, 2009 and, in revised form, November 10, 2009.

2000 Mathematics Subject Classification. Primary 05E05; Secondary 05E10, 33D52.

Key words and phrases. Key polynomials, non-symmetric Macdonald polynomials, LittlewoodRichardson rule, quasisymmetric functions, Schur functions, tableaux.

(C)2010 American Mathematical Society 1665

Reverts to public domain 28 years from publication 
of the nice analytic and algebraic properties of the $J_{\mu}$. Macdonald showed how to express $J_{\mu}$ as a linear combination of the $E_{\gamma}$, which can thus be thought of as more fundamental building blocks. Macdonald's definition of the $E_{\gamma}$ was rather indirect, but in [5] a new combinatorial formula for the (type A) $E_{\gamma}$ was introduced. By letting $q=t=0$ and $q=t=\infty$ in this formula we obtain new combinatorial formulas for Demazure characters (first studied by Demazure in [2]) and Demazure atoms (called standard bases by Lascoux and Schützenberger [11]), respectively. These formulas are described in terms of skyline fillings, which are combinatorial objects related to tableaux. Mason [15], [16] showed that many of the interesting properties of Demazure characters and atoms can be explained via the combinatorics of skyline fillings. In particular she developed a refinement of the well-known Robinson-Schensted-Knuth algorithm, involving skyline fillings and weak compositions, which shows bijectively that the Schur function $s_{\lambda}\left(X_{n}\right)$ is a sum of those atoms corresponding to weak compositions $\gamma$ with $n$ parts whose non-zero parts are a rearrangement of the parts of $\lambda$.

One natural question to ask is how this decomposition of $s_{\lambda}$ into atoms compares with the well-known fact [22, p. 361] that $s_{\lambda}$ is a sum, over standard Young tableaux $T$ of shape $\lambda$, of Gessel's fundamental quasisymmetric function $F_{\operatorname{des}(T)}$. In [6] the authors showed that, if $\gamma^{+}$is the composition obtained by removing all zero parts from $\gamma$ (so, for example, $100203401^{+}=12341$ ), then the sum of Demazure atoms, over all $\gamma$ with $\gamma^{+}$equaling a fixed composition $\beta$, is a sum of certain fundamental quasisymmetric functions, and hence also quasisymmetric. We call this sum the quasisymmetric Schur function (QS for short), denoted $\mathcal{S}_{\beta}\left(X_{n}\right)$, and note that $s_{\lambda}\left(X_{n}\right)$ is the sum, over all compositions $\beta$ whose parts are a rearrangement of the parts of $\lambda$ (denoted $\tilde{\beta}=\lambda$ ), of $\mathcal{S}_{\beta}\left(X_{n}\right)$. In general there are fewer terms in this expansion than the expansion into Gessel's $F$ 's; for example, if $\lambda$ is a rectangle, then there is only one multiset permutation of the parts of $\lambda$, and hence $s_{\lambda}=\mathcal{S}_{\lambda}$.

The family of QS functions forms a new basis for the ring of quasisymmetric functions. Although the product of two fundamental quasisymmetric functions expands as a positive sum of fundamental quasisymmetric functions [4, it turns out that the product of two QS functions does not expand as a positive sum of QS functions. In [6] the authors showed though that if you multiply a QS function by either a complete homogeneous symmetric function or an elementary symmetric function, the result is a positive sum of QS functions, which can be thought of as a version of the famous Pieri rule. The current investigation grew out of an observation of the authors that the product of a Schur function and a QS function is a positive sum of QS functions. Efforts to understand the coefficients in this expansion combinatorially led to the discovery that the product of a Schur function and a Demazure atom has a positive expansion into atoms, and that the coefficients in this expansion can be described in terms of analogues of Littlewood-Richardson tableaux (also known as Yamanouchi tableaux), in the context of skyline fillings. We prove this in Section 4. borrowing many ideas contained in the proof in Fulton's book [3] of the classical Littlewood-Richardson rule, replacing statements about semistandard Young tableaux (SSYT) by corresponding statements about skyline fillings. In Sections 5 and 6 we show how our Littlewood-Richardson rule for atoms leads to corresponding rules for both QS functions and Demazure characters. Since Schur functions are special cases of Demazure characters, we obtain the classical Littlewood-Richardson rule as a special case. Note that in 18, Reiner and Shimizono obtain a number of 

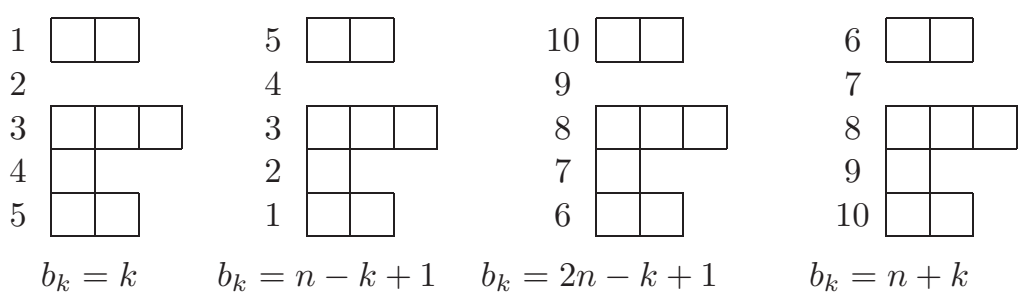

Figure 1. Skyline diagrams with $n=5$, composition $(2,0,3,1,2)$, and four typical basements

results involving the expansion of various generalizations of skew Schur functions as a positive sum of Demazure characters, which yield identities similar in spirit to our expansion of the product of a Schur function and a Demazure character.

Every Schubert polynomial can be written as a positive sum of type A Demazure characters. This means that our results provide a method for expanding the product of an arbitrary Schubert polynomial and Schur function (in the same set of variables) as a positive sum of Demazure characters. Combinatorial descriptions of the coefficients that arise in the product of a Schur polynomial and certain Schubert polynomials when expanded as a sum of Schubert polynomials are given in [8] and 9]. Their proofs involve concepts that are similar to ones we use, and one wonders whether it is possible to recover these results, or generalizations of them, using our techniques. One also wonders whether there is an underlying structure unifying our positivity expansions and whether this structure is related to other structures underlying algebraic positivity, for example, Polo's notion of $B$ modules with excellent filtration [17, 7].

\section{BASIC DEFINITIONS AND NOTATION}

2.1. Skyline diagrams. A skyline diagram is a collection of boxes, or cells, arranged into left-justified rows 11 To each skyline diagram we associate a weak composition, whose $k$-th part is the number of cells in the $k$-th row of the diagram, where the top row is viewed as row 1, the row below it row 2, et cetera. Skyline diagrams are augmented by a basement, an extra column on the left (considered to be the 0 -th column) containing positive integers. We let $b_{k}$ denote the entry in the $k$-th row of the basement. In most of our examples the basement will either satisfy $b_{k}=k, b_{k}=n-k+1, b_{k}=n+k$, or $b_{k}=2 n-k+1$ for $1 \leq k \leq n$, as in the diagrams in Figure 1 .

Let $\gamma, \delta$ be weak compositions with $\gamma \subseteq \delta$, i.e. $\gamma_{i} \leq \delta_{i}$ for $1 \leq i \leq n$.

A skew skyline diagram of shape $\delta / \gamma$ is obtained by starting with a skyline diagram of shape $\delta$ with basement values $\left(b_{1}, b_{2}, \ldots, b_{n}\right)$ and by considering the cells of $\gamma$ to be an extension of the basement, placing the value $b_{k}$ in each of the cells in the $k$-th row of $\gamma$. A skyline diagram of shape $\delta$ can naturally be viewed as a skew skyline diagram of shape $\delta /(0,0, \ldots, 0)$. Note that if the parts of $\delta$ and of $\gamma$ are monotone decreasing, and we remove the basement, we get a skew Ferrers shape.

\footnotetext{
${ }^{1}$ This differs slightly from the convention in [5, [15, 16], where skyline diagrams are arranged in bottom-justified columns.
} 
A skyline filling (skew skyline filling) is an assignment of positive integers to the cells of a skyline (skew skyline) diagram, respectively. Central to our constructions involving skyline fillings is a triple of cells, of which there are two types. A type A triple in a diagram of shape $\delta / \gamma$ is a set of three cells $a, b, c$ of the form $(i, k),(j, k),(i, k-1)$ for some pair of rows $i<j$ of the diagram and some column $k>0$, where row $i$ is at least as long as row $j$, i.e. $\delta_{i} \geq \delta_{j}$. A type $B$ triple is a set of three cells $a, b, c$ of the form $(j, k+1),(i, k),(j, k)$ for some pair of rows $i<j$ of the diagram and some column $k \geq 0$, where row $i$ is strictly shorter than row $j$, i.e. $\delta_{i}<\delta_{j}$. Note that basement cells can be elements of triples. As noted below, in this article our fillings have weakly decreasing row entries left to right, so we always have the entry values $c \geq a$. We say that a triple of either type is an inversion triple if the relative order of the entries is either $b<a \leq c$ or $a \leq c<b$. Otherwise we say that the triple is a coinversion triple, i.e. $a \leq b \leq c$.

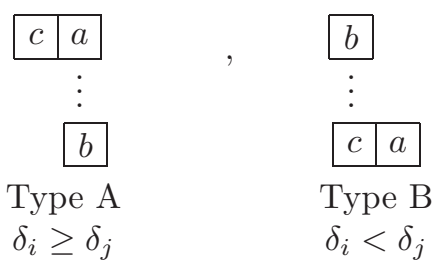

A semistandard skyline filling (SSK) is a (skew) skyline filling where

(i) each row is weakly decreasing left to right (including the basement) and

(ii) all triples (including triples with cells in the basement) are inversion triples.

Remark 2.1. Note that since basement values are constant across rows, for any choice of basement values any triple involving three basement cells is forced to be an inversion triple. Furthermore, if we have a skew skyline diagram with basement $b_{k}=2 n-k+1$, all entries in the basement are larger than $n$, the biggest entry outside the basement. Therefore, the actual values of the $b_{k}$ are not relevant, as long as they are decreasing from top to bottom and all are larger than $n$. For this reason we often draw the basement $b_{k}=2 n-k+1$ with "*" symbols in place of the $b_{k}$, where we think of the $*$ as a virtual $\infty$ symbol, larger than any entry, and we refer to this basement as the large basement. To determine whether a triple involving two $*$ symbols is an inversion triple or not, we view $*$ symbols in the same row as being equal, and $*$ symbols in a given column as decreasing from top to bottom. In our identities involving the large basement and polynomials depending on $X_{n}$, we can let $n \rightarrow \infty$ and view the identity as holding in the infinite set of variables $X=\left\{x_{1}, x_{2}, \ldots\right\}$.

Figure 2 gives examples of SSK for various shapes $\delta / \gamma$. The portion of the basement whose cells are part of $\gamma$ are indicated by light gray backgrounds. It is shown in 15] that every SSK is non-attacking, meaning that the entries within each column are all distinct and that two cells $a=(i, k)$ and $b=(j, k+1)$ can only have the same value if $i \geq j$.

2.2. Contretableaux and reading words. A contretableau (CT) is a Ferrers shape filled with positive integers where the entries within each row decrease weakly left to right and the entries within each column decrease strictly top to bottom. We let $\mathrm{CT}(n)$ denote the set of $\mathrm{CT}$ with entries from the set $[n]=\{1,2, \ldots, n\}$. Note 

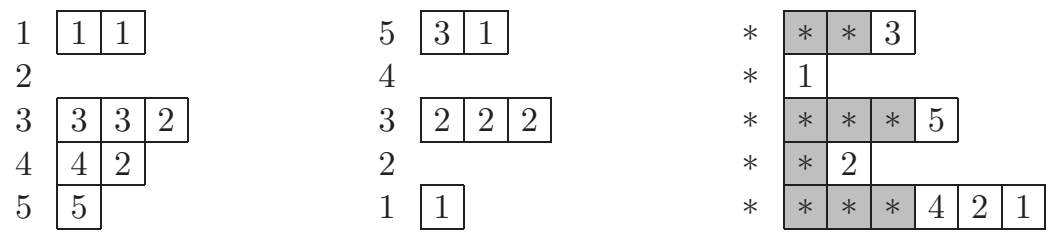

Figure 2. SSK of shapes $(2,0,3,2,1),(2,0,3,0,1)$, and $(3,1,4,2,6) /(2,0,3,1,3)$

that $\operatorname{CT}(n)$ is trivially in bijection with $\operatorname{SSYT}(n)$, the set of $\operatorname{SSYT}$ with entries from $[n]$, by applying the map $j \rightarrow n-j+1$ to each entry of a given CT.

Since CT are trivially equivalent to SSYT, it is no surprise that all of the concepts, definitions, operations (such as insertion and evacuation), propositions, and theorems regarding SSYT have CT-counterparts, and the proofs of such results are completely analogous. We include in this section several of the classical notions most pertinent to our results; the (SSYT versions of the) fully developed theory can be found in [3] or [22].

The row reading order of a (possibly skew) skyline diagram or Ferrers shape is a total ordering of the cells where $(i, j)<_{\text {row }}\left(i^{\prime}, j^{\prime}\right)$ if either $i>i^{\prime}$ or $\left(i=i^{\prime}\right.$ and $\left.j<j^{\prime}\right)$. That is, the row reading order reads the cells left to right in each row, starting with the bottommost row and proceeding upwards to the top row, ignoring basement entries if they exist. The row word of a filling $T$, denoted $\operatorname{rowword}(T)$ is the sequence of integers formed by the entries of $T$ taken in row reading order.

We also use a slightly different reading order on diagrams, which we refer to as the column reading order. In the column reading order, we have $(i, j)<_{\text {col }}\left(i^{\prime}, j^{\prime}\right)$ if either $j>j^{\prime}$ or $\left(j=j^{\prime}\right.$ and $\left.i<i^{\prime}\right)$. That is, the column reading order reads the cells from top to bottom within each column, starting with the rightmost column and working leftwards, again ignoring any basement entries. The column word of a filling $T$, denoted colword $(T)$, is the sequence of integers formed by the entries of $T$ taken in column reading order. For example, for the rightmost SSK in Figure 2, the row word is 4212513 and the column word is 1254321 .

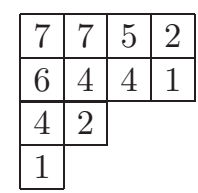

CT

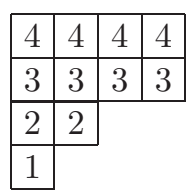

the super CT

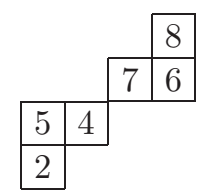

skew CT on $(4,4,2,1) /(3,2)$

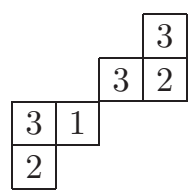

LR CT of content $(1,2,3)$

Figure 3. CT examples

Definition 2.2. For a word $w=w_{1} w_{2} \cdots w_{n}$ (or sequence $\left.\left(w_{1}, \ldots, w_{n}\right)\right)$ we let $w^{*}$ denote the reverse word $w_{n} w_{n-1} \cdots w_{2} w_{1}$ (or sequence $\left(w_{n}, \ldots, w_{1}\right)$ ). The content of $w$ is the sequence $\left(c_{1}, c_{2}, \ldots, c_{n}\right)$, where $c_{i}$ is the number of occurrences of $i$ in $w$. The content of a CT $T$ is the content of $\operatorname{colword}(T)$.

For a partition $\lambda$ with $r$ parts, the super $C T$ of shape $\lambda$ (denoted $U_{\lambda}$ ) is the unique CT with content $\lambda^{*}$, as in Figure 3, For the "contre-" analog $V \leftarrow W$ of 
inserting/recording a biword $W$ into a CT $V$, the biletters of $W$ are sorted into reverse (i.e. weakly decreasing) lexicographical order.

A lattice word is a word (or sequence) $w=w_{1} w_{2} \cdots w_{n}$ where in any initial segment $w_{1} w_{2} \cdots w_{i}$ there are at least as many occurrences of the number $j$ as $j+1$, for each $j \geq 1$. We say $w$ is contre-lattice if in any initial segment there are at least as many occurrences of the number $j$ as $j-1$, for each $1<j \leq r$, where $r$ is the maximum of the $w_{i}$. We say a word or sequence $w$ is regular contrelattice if it is contre-lattice and the minimum of the $w_{i}$ is 1 . We define a Littlewood Richardson skew $C T$ to be a skew CT the reverse of whose row reading word is regular contre-lattice. We often abbreviate "Littlewood-Richardson" by "LR" .

Proposition 2.3 (3. Section 5.2]). Let $S$ be a skew CT with content $\mu^{*}$. Then the following are equivalent:

(i) $S$ is an $L R$ skew $C T$, i.e. rowword $(S)^{*}$ is a regular contre-lattice word.

(ii) $\operatorname{colword}(S)$ is a regular contre-lattice word.

(iii) $\operatorname{rect}(S)=U_{\mu}$, the super $C T$ of shape $\mu$. (Here $\operatorname{rect}(S)$ is the "rectification" of S; see [3].)

2.2.1. Combinatorial formulas. Recall the well-known combinatorial formula for the Schur function

$$
s_{\lambda}=\sum_{\substack{T \in \operatorname{SSYT}(n), \\ \text { shape }(T)=\lambda}} \mathbf{x}^{T}
$$

The following combinatorial formulas for Demazure atoms $\mathcal{A}_{\gamma}$ and Demazure characters $\kappa_{\gamma}$ follow as limiting cases of results in [5]:

$$
\begin{aligned}
\mathcal{A}_{\gamma} & =\sum_{\substack{Y \in \operatorname{SSKI}(n), \\
\text { shape }(Y)=\gamma}} \mathbf{x}^{Y}, \\
\kappa_{\gamma} & =\sum_{\substack{Y \in \operatorname{SSKD}(n), \operatorname{shape}(Y)=\gamma^{*}}} \mathbf{x}^{Y},
\end{aligned}
$$

where $\operatorname{SSKI}(n)$ is the set of all SSK with basement $b_{k}=k$ and entries in $[n]$, and $\operatorname{SSKD}(n)$ is the set of all SSK with entries in $[n]$ and $b_{k}=n-k+1$.

Taylor [23], building on the work of [1, 10, [19, 20, 21, describes a class of generalized tableaux over signed alphabets which he calls straight. Straight tableaux have overall partition shape and include the classical skew SSYT for allpositive alphabets. They are defined in part by using a triple condition on cells which, if we reverse his inequalities and consider only positive alphabets, would be the same as our type A inversion triple condition. Additionally, those SSKD with partition shape are equivalent to CT and thus to SSYT, which are special cases of straight tableaux. It would be interesting to know whether there is a more significant relationship between Taylor's straight tableaux and the objects in this article. 
2.2.2. A bijection between $\operatorname{SSKI}(n)$ and $C T(n)$. There exists a simple bijection $\rho$ between $\operatorname{SSKI}(n)$ and $\operatorname{CT}(n)$ [15. Given $Y \in \operatorname{SSKI}(n)$, one obtains the corresponding CT by sorting the entries within each column, as in the example below:

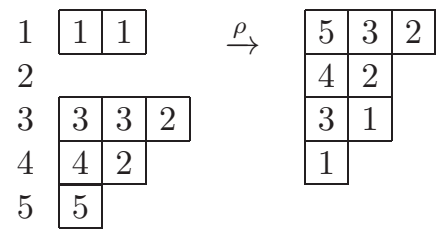

The inverse $\rho^{-1}$ is only slightly more intricate. Given $T \in \mathrm{CT}(n)$, map the leftmost column of $T$ into an empty element of $\operatorname{SSKI}(n)$ by placing the entries of this column, beginning with the largest, into the highest row of the leftmost column whose rightmost entry is weakly greater. Repeat this procedure with each of the remaining columns. One important property to note about the bijection is that it preserves the set of entries within each column. (We say set as opposed to multiset since all of our tableau-like structures require that all entries within a column be distinct.)

2.2.3. Pieri rules. Pieri rules for multiplying a QS function by a complete homogeneous symmetric function $s_{k}$ or an elementary symmetric function $s_{1^{k}}$ are presented in [6]. By the same method one can derive Pieri rules for multiplying a Demazure atom by either $s_{k}$ or $s_{1 k}$. The intersecting case $s_{1}$, the "single box case", can be described as follows. Given a weak composition $\delta$ containing a part with value $k$, $k>0$, define $\operatorname{rem}_{k}(\delta)$ to be the weak composition resulting from decrementing the last (rightmost) part of $\delta$ that has value $k$. For example,

$$
\operatorname{rem}_{2}(1,0,4,2,0,1,2,3)=(1,0,4,2,0,1,1,3) .
$$

We likewise define $\operatorname{rem}_{k}(\beta)$ for compositions, where the result is collapsed to a composition by removing any resulting zero part. For example,

$$
\text { rem }_{1}(1,4,2,1,2,3)=(1,4,2,2,3) .
$$

Now the "single box" Pieri rule can be described as

$$
\begin{aligned}
& \mathcal{A}_{\gamma}\left(X_{n}\right) \cdot s_{1}\left(X_{n}\right)=\sum_{\delta} \mathcal{A}_{\delta}\left(X_{n}\right), \\
& \mathcal{S}_{\alpha}\left(X_{n}\right) \cdot s_{1}\left(X_{n}\right)=\sum_{\beta} \mathcal{S}_{\beta}\left(X_{n}\right),
\end{aligned}
$$

where $\delta$ runs over all weak compositions satisfying $\gamma=\operatorname{rem}_{k}(\delta)$ for some positive integer $k$, and similarly $\beta$ runs over all compositions satisfying $\alpha=\operatorname{rem}_{k}(\beta)$ for some positive integer $k$. Note the close similarity between these rules and the corresponding rule for Schur functions [22, p. 337].

\section{Properties OF SKYLINE FILLINGS}

For a given cell $x$ in a skyline diagram, we let row $(x)$ denote the row containing $x$. Say that an SSK $Y$ on any basement is contre-lattice if its column reading word is contre-lattice. Suppose $Y$ is an SSK on any basement, where $Y$ has $t$ columns. For all $1 \leq k \leq t$, let $C_{k}$ be the set of entries in column $k$ of $Y$, excluding basement entries. Call these the column sets of $Y$. Sort each $C_{k}$ into decreasing order and 
form the word $w_{Y}=C_{t} C_{t-1} \cdots C_{2} C_{1}$. We say that $Y$ is loosely contre-lattice if $w_{Y}$ is contre-lattice.

Proposition 3.1. Let $Y$ be an element of $\operatorname{SSK}(n)$ on any basement satisfying $b_{k}>n$ for $1 \leq k \leq n$. Then $Y$ is contre-lattice if and only if $Y$ is loosely contrelattice.

Proof. Assume $Y$ is contre-lattice and $Y$ has $t$ columns. Let $C_{k}^{\prime}$ be the sequence of the elements of the $k$-th column of $Y$ in column reading order, so that $\operatorname{colword}(Y)=$ $C_{t}^{\prime} C_{t-1}^{\prime} \cdots C_{2}^{\prime} C_{1}^{\prime}$. By assumption, $\operatorname{colword}(Y)$ is contre-lattice. If within this word we transpose any adjacent pair $w_{i} w_{j}$ of letters in the word, where $w_{i}<w_{j}$, then the resulting word retains the contre-lattice property. In particular, if we sort each of the $C_{k}^{\prime}$ into decreasing order to obtain $C_{k}$, the resulting word $w_{Y}=C_{t} C_{t-1} \cdots C_{2} C_{1}$ retains the contre-lattice property. Thus $Y$ is loosely contre-lattice.

Conversely, assume that $Y$ is loosely contre-lattice. Label the cells of $Y$ according to their contents and place them in the column reading order of $Y$. Specifically, we identify a cell of $Y$ as $x_{j}$ when the cell contains the $j$-th occurrence of the entry $x$ in $\operatorname{colword}(Y)$. Let $m$ be the number of $r$ 's in $\operatorname{colword}(Y)$, and for each $1 \leq k \leq m$ let $S_{k}=\left\{x_{k}: 1 \leq x \leq r, x_{k} \in Y\right\}$. To show that $Y$ is contre-lattice, it suffices to show that for each $k$, the cells of $S_{k}$, as they appear in the column reading order of $Y$, are in strictly decreasing order of their contents. Since $Y$ is loosely contre-lattice, if two cells of $S_{k}$ are in different columns of $Y$, then they appear in the column reading order of $Y$ in strictly decreasing order of their contents. Thus it suffices to show that if two cells of $S_{k}$ lie in the same column of $Y$, then they also appear in strictly decreasing order of their contents. That is, we need to show that if $x<y$ and $x_{k}, y_{k} \in S_{k}$ are in the same column, then $y_{k}$ appears above $x_{k}$ in that column.

Seeking a contradiction, suppose this is not the case. Among all such violating pairs of values, choose $x$ and $y$ such that $|y-x|$ is minimized.

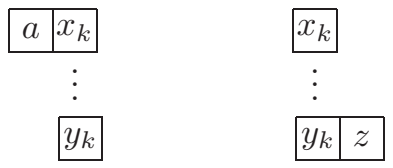

We consider two cases. In the first case, $\operatorname{row}\left(x_{k}\right)$ is at least as long as row $\left(y_{k}\right)$, corresponding to the first diagram above, where $a$ is the entry immediately to the left of $x_{k}$. Without loss of generality, we may assume that $k$ is the largest among such indices for this case. Since $Y$ has no coinversion triples, it must be the case that $x_{k} \leq a<y_{k}$, implying that $a$ is not in the basement. If $a=x$, then the cell with entry $a$ shown is in fact $x_{k+1}$. This implies that $y_{k+1}$ is also in the same column as $x_{k+1}$. Since $Y$ is non-attacking, $y_{k+1}$ must appear weakly below $y_{k}$, and hence below $x_{k+1}$, contrary to the assumption that $k$ is maximal. Thus $x_{k}<a<y_{k}$. This in turn implies that $a_{k}$ is in the same column as $x_{k}$ and $y_{k}$ and also that $a=a_{k+1}$. Since $Y$ is non-attacking, $a_{k}$ must appear weakly above $a_{k+1}$, and hence above $y_{k}$. But then $y$ and $a$ form a violating pair with $|y-a|<|y-x|$, contrary to our assumption that $|y-x|$ is minimal. Thus we have a contradiction in this case.

The other case is that row $\left(x_{k}\right)$ is shorter than row $\left(y_{k}\right)$, corresponding to the second diagram above, where $z$ is the entry immediately to the right of $y_{k}$. Without loss of generality, we may assume that $k$ is the smallest among such indices for this case. Since $Y$ has no coinversion triples, it must be the case that $x_{k}<z \leq y_{k}$. If $z=y$, then the cell with entry $z$ shown is in fact $y_{k-1}$. This implies that $x_{k-1}$ is also 
in the same column as $y_{k-1}$. Since $Y$ is non-attacking, $x_{k-1}$ must appear weakly above $x_{k}$, and hence above $y_{k-1}$, contrary to the assumption that $k$ is minimal. Thus $x_{k}<z<y_{k}$. This in turn implies that $z_{k}$ is in the same column as $x_{k}$ and $y_{k}$ and also that $z=z_{k-1}$. Since $Y$ is non-attacking, $z_{k}$ must appear below $z_{k-1}$, and hence below $x_{k}$. But then $x$ and $z$ form a violating pair with $|z-x|<|y-x|$, contrary to our assumption that $|y-x|$ is minimal. Thus we have a contradiction in this case. Thus in all cases we obtain a contradiction, which completes the proof.

Proposition 3.2. Let $Y$ be a contre-lattice element of $S S K(n)$ on a decreasing (i.e. $b_{i}>b_{i+1}$ for $1 \leq i<n$ ) basement. Let $x$ be the smallest entry value in $Y$, and let $x_{1}$ be the cell containing the rightmost entry of value $x$, i.e. the first $x$ in column reading order. Then $x_{1}$ is the rightmost cell of its row, say row $i$, and for every row $i^{\prime}>i$, row $i$ and row $i^{\prime}$ have different lengths.

Proof. That $x_{1}$ is at the end of its row is immediate since $x$ is the smallest entry value in $Y$. Suppose that there is some row $i^{\prime}>i$ of the same length as row $i$. Let $z$ be the entry in the last cell of row $i^{\prime}$.

$$
\begin{array}{lll|l|l|l|}
b & \cdots & u & v & \cdots & x_{1} \\
b^{\prime} & \cdots & w & y & \cdots & z \\
\hline
\end{array}
$$

Since $x$ is the smallest entry value in $Y$, we have $x<z$. On the other hand, since the basement is decreasing, the basement entries for the rows are related by $b>b^{\prime}$, where $b=b_{i}=Y(i, 0)$ and $b^{\prime}=b_{i^{\prime}}=Y\left(i^{\prime}, 0\right)$. Thus there must exist some column $j$ such that $v<y$ and $u>w$, where $v=Y(i, j+1), y=Y\left(i^{\prime}, j+1\right), u=Y(i, j)$, and $w=Y\left(i^{\prime}, j\right)$. This implies that $v<y<u$, which would form a type A coinversion triple, a contradiction. Thus there can be no such row $i^{\prime}$.

Proposition 3.3. Let $Y$ be a contre-lattice element of $S S K(n)$ on any basement. Let $x$ be the smallest entry value in $Y$, and let $x_{1}$ be the cell containing the rightmost entry of value $x$, i.e. the first $x$ in column reading order. Then the skyline diagram filling $Y^{\prime}=Y-x_{1}$ obtained from $Y$ by simply removing cell $x_{1}$ is also a contrelattice SSK.

Proof. As above, assume $x_{1}$ is in row $i$. Since $Y$ is already an SSK, to show that $Y^{\prime}$ is an SSK, it suffices to show that removing $x_{1}$ does not introduce any coinversion triples, which could only happen between row $i$ and some other row $i^{\prime}$. No type B conversion triples could be introduced since by Proposition 3.2 there are no rows in $Y$ below row $i$ of the same length as row $i$. Any type A coinversion triples introduced would have to be between a row $i^{\prime}<i$ of length one less than that of row $i$ in $Y$. Suppose that such a conversion triple $u, v, w$ exists in $Y^{\prime}$ between rows $i^{\prime}$ and $i$, as shown:

$$
\begin{aligned}
& \cdots \begin{array}{|l|l|l|l|l|}
\hline w & u & \cdots & a & c \\
\hline
\end{array} \\
& \begin{array}{lll|l|l|l|l|}
\cdots & v & \cdots & b & d & \cdots & z \\
\hline
\end{array}
\end{aligned}
$$

The relation between these values must be $u<v<w$. In particular, $u<v$. On the other hand, the triple $x_{1}, y, z$ occurring at the end of rows $i$ and $i^{\prime}$ in $Y$, as shown ( $z$ is to the immediate left of $x_{1}$ ), must be an inversion triple, and since $x$ is the smallest entry value in $Y$, this implies the order $x \leq z<y$. In particular, 
$y>z$. Thus there must exist some column $j$ such that $a<b$ and $c>d$, where $a=Y\left(i^{\prime}, j\right), b=Y(i, j), c=Y\left(i^{\prime}, j+1\right)$, and $d=Y(i, j+1)$. This implies that $d<c \leq a<b$. In particular, $d, a, b$ would form a type $\mathrm{B}$ coinversion triple in $Y$, a contradiction. Thus there can be no such type A coinversion triple in $Y^{\prime}$, and so $Y^{\prime}$ is an SSK.

Lastly, removing the first occurring smallest-value letter from a contre-lattice word clearly leaves another contre-lattice word, and so $Y^{\prime}$ is also contre-lattice.

Proposition 3.4. Let $Y$ be a contre-lattice element of $S S K(n)$ of shape $\delta / \gamma$ on any basement of shape $\gamma$. Let $\sigma$ be any permutation of $\delta$. Then there exists a unique contre-lattice SSK T of overall shape $\sigma$ on a large basement $b_{i}=2 n-i+1$ having the same column sets as $Y$. Moreover, $T$ has shape $\sigma / \tau$ for some basement shape $\tau$ that is a permutation of $\gamma$.

Proof. We proceed by induction on the number of cells in $Y$, that is, $|\delta / \gamma|$. Start with the unfilled skyline diagram of shape $\sigma$. Let $x$ be the smallest entry value in $Y$, and let $j$ be the column of the rightmost occurrence of $x$ in $Y$. Since $x$ is the smallest entry value, it occurs at the end of some row of $Y$ of length $j$. Proposition 3.2 tells us that if $T$ exists, then the entry $x$ in column $j$ of $T$ must occur in the last row of $T$ of length $j$, say row $i$, which exists since $\sigma$ is a permutation of $\delta$. Let $Y^{\prime}$ be the SSK obtained by removing the cell in column $j$ of $Y$ that contains $x$. Let $\sigma^{\prime}$ be the shape obtained by removing the last cell of the last row of length $j$ in $\sigma$. If $|\delta / \gamma|=1$, we are done; we set the basement of $T$ to be of shape $\sigma^{\prime}$.

Otherwise, by Proposition 3.3, $Y^{\prime}$ is also a contre-lattice SSK, say of shape $\delta^{\prime} / \gamma$, and clearly $\sigma^{\prime}$ is a permutation of $\delta^{\prime}$. By our induction hypothesis there is a unique contre-lattice SSK $T^{\prime}$ of overall shape $\sigma^{\prime} / \tau$ on a large basement $b_{i}=2 n-i+1$ having the same column sets as $Y^{\prime}$, where $\tau$ is a permutation of $\gamma$. We want to show that if we append a cell containing $x$ to row $i$ of $T^{\prime}$, which must be in column $j$, then the resulting filling $T$ is an SSK. Since $x$ was the minimum entry of $Y$ and column $j$ its rightmost appearance, the cell added to $T^{\prime}$ to form $T$ is the rightmost minimum entry of $T$. In particular, it is less than or equal to the entry to its immediate left, so row $i$ of $T$ is weakly decreasing, as are all other rows of $T$.

It remains to check the triple conditions. Consider row $i^{\prime}$, where $i^{\prime} \neq i$. If the relative order of the lengths of the two rows $i$ and $i^{\prime}$ is unchanged when comparing $T^{\prime}$ to $T$, then the type of triples between the two rows remains the same, and we only need consider any new triple formed by adding the new cell. In any new triple formed, $x$ lies in row $i$ while the other two cells of the triple lie in row $i^{\prime}$, and since $x$ is the rightmost occurrence of the minimum value in $T$, it cannot form a coinversion triple between the two rows.

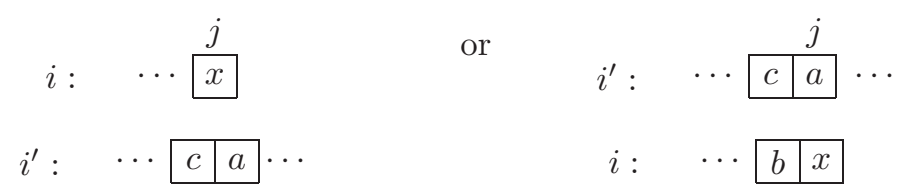

The only remaining case occurs when $i^{\prime}<i$ and $\sigma_{i^{\prime}}^{\prime}=\sigma_{i}^{\prime}$, when $\sigma_{i^{\prime}}<\sigma_{i}$. In this case, whereas $T^{\prime}$ had type A triples between the two rows, now $T$ has type B triples between them. Suppose that one of these type B triples in $T$ is a coinversion triple, say $v, w, u$ as in the diagram below, where $v$ and $w$ are in column $j^{\prime}$, and 
where possibly the cell $u$ is the cell at the end of row $i$.

$$
\begin{aligned}
& j^{\prime} \quad j \\
& \begin{array}{lll|l|l|l|l|l|l|l|l}
i^{\prime}: & b^{\prime} & \cdots & a & c & \cdots & \cdots & \cdots & y
\end{array}
\end{aligned}
$$

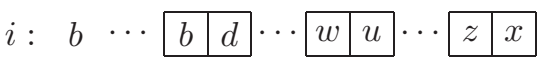

This requires that $u \leq v<w$. On the other hand, since $T$ and $T^{\prime}$ share a common decreasing basement, the basement entries in these rows satisfy $b^{\prime}>b$. This implies that there exists some pair of adjacent columns in the range 0 to $j^{\prime}$ inclusive containing the cells $a, b, c$, and $d$ of the two rows as shown such that $a>b$ and $c<d$. But that would imply that $c<d<a$, forming a type A coinversion triple in $T^{\prime}$, contrary to the fact that $T^{\prime}$ is a valid SSK. Thus all the type B triples between the two rows in $T$ are inversion triples. In all cases, $T$ is a valid SSK.

By Proposition 3.1. $Y$ is loosely contre-lattice. Since $T$ has the same column sets as $Y, T$ is therefore also loosely contre-lattice, and again by Proposition 3.1 $T$ is contre-lattice.

Remark 3.5. The proof of Proposition 3.4 provides us with an algorithm for constructing the desired SSK on a large basement by successively filling the "lowest" row strip in the unfilled portion of the diagram for the set of columns containing the smallest-valued entries at each step, as illustrated in Figure 4. An easy argument shows that starting with an SSK $Y$ as in the statement of the proposition, if we have two compositions $\sigma$ and $\sigma^{\prime}$, both permutations of $\delta$ with $\sigma^{+}=\sigma^{\prime+}$, then the respective constructed SSK $L$ and $L^{\prime}$ will have respective shapes $\sigma / \tau$ and $\sigma^{\prime} / \tau^{\prime}$ with $\tau^{+}=\tau^{\prime+}$.

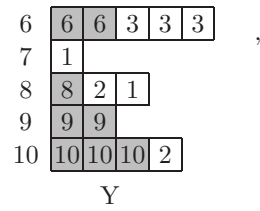

$(5,3,2,4,1)$

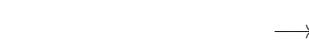

$\sigma$

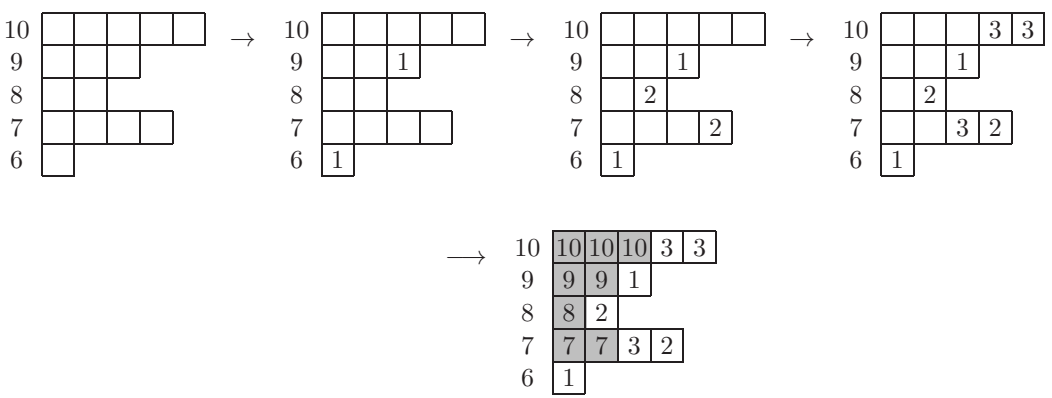

Figure 4. Construction example for a pair $Y, \sigma$ 


\section{Littlewood-Richardson Rule for Demazure atoms}

A Littlewood-Richardson skew skyline tableau (LRS) of shape $\delta / \gamma$ is an SSK of shape $\delta / \gamma$ with large basement $b_{i}=2 n-i+1$, where $n=\ell(\delta)=\ell(\gamma)$, whose column reading word is a regular contre-lattice word. Figure 5 shows an example of an LRS with column reading word 3231321, which is regular contre-lattice of content $(2,2,3)$. We let $\operatorname{LRS}(n)$ denote the set of LRS with entries in $[n]$.

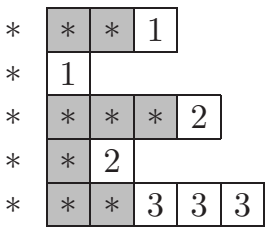

FIGURE 5. An LRS with $n=5$ and column reading word 3231321

We can now state our LR rule for the product of a Schur function and a Demazure atom.

Theorem 4.1. In the expansion

$$
\mathcal{A}_{\gamma}\left(X_{n}\right) \cdot s_{\lambda}\left(X_{n}\right)=\sum_{\delta} a_{\gamma \lambda}^{\delta} \mathcal{A}_{\delta}\left(X_{n}\right),
$$

the coefficient $a_{\gamma \lambda}^{\delta}$ is the number of elements in $L R S(n)$ of shape $\delta / \gamma$ with content $\lambda^{*}$.

Proof. As with the proof of the classical LR rule for Schur functions [3], we recall the homomorphism $\psi: T \mapsto \mathrm{x}^{T}$ from the contretableau ring $R_{n}$, the graded algebra whose basis is $\operatorname{SSYT}(n)$, onto the polynomial $\operatorname{ring} \mathbb{Z}[X]=\mathbb{Z}\left[x_{1}, \ldots, x_{n}\right]$. Under the bijection $\rho$ we may identify SSK with their corresponding CT. The combinatorial formulas given in equations (2.1) and (2.2) allow us to identify pre-images of Schur functions and Demazure atoms:

$$
\begin{array}{rlrl}
S_{\lambda} & =\sum_{\substack{V \in \operatorname{CT}(n), \operatorname{shape}(V)=\lambda}} V, & \psi\left(S_{\lambda}\right) & =s_{\lambda}\left(X_{n}\right), \\
A_{\gamma}= & \sum_{\substack{U \in \operatorname{SSKI}(n), \\
\text { shape }(U)=\gamma}} U, & \psi\left(A_{\gamma}\right)=\mathcal{A}_{\gamma}\left(X_{n}\right) .
\end{array}
$$

Under the homomorphism we then have $\psi\left(A_{\gamma} \cdot S_{\lambda}\right)=\mathcal{A}_{\gamma}\left(X_{n}\right) \cdot s_{\lambda}\left(X_{n}\right)$. The terms of $A_{\gamma} \cdot S_{\lambda}$ are the products of ordered pairs of CT $(U, V)$ where $\rho^{-1}(U)$ has shape $\gamma$. The idea of the proof is then to exploit the bijection $(U, V) \leftrightarrow(T, S)$ between ordered pairs $(U, V)$ of arbitrary $\mathrm{CT}$ and pairs $(T, S)$ of a $\mathrm{CT} T=U \cdot V$ and a recording LR skew CT $S$, restricting ourselves to the case where the image $\rho^{-1}(U)$ has shape $\gamma$.

The bijection matches the CT $V$ (which here has shape $\lambda$ ) with a super CT of the same shape, and the pair is mapped to a biword $W$ using the RSK correspondence. (See [22, Chapter 7] for a discussion of the RSK algorithm. Note that for CT, the biword $W$ is in reverse lexicographic order.) We then compute $(T, S)=U \leftarrow W$. 
That is, the lower row of the biword is inserted into $U$ to obtain the pair $T=U \cdot V$, while the upper row of the biword is placed into the corresponding skew Ferrers shape to obtain an LR skew CT $S$. In the same way we can compute $\left(\rho^{-1}(T), L\right)=$ $\rho^{-1}(U) \leftarrow W$. As we insert/place the biletters one by one, we can also track the images of the intermediate $\mathrm{CT}$ under the bijection $\rho^{-1}$; that is, inserting the bottom row of $W$ into the SSK $\rho^{-1}(U)$ using the insertion map described in [15] and placing the upper row of $W$ in an SSK $L$, recording the location of the new cell. Figure 6 gives an example. The resulting insertion SSK will of course be $\rho^{-1}(T)$, say of shape $\delta$. It remains to show that (1) the resulting SSK $L$, when combined with the basement $b_{i}=2 n-i+1$, is in fact an LRS, and (2) conversely, that any LRS $L$ of shape $\delta / \gamma$ and weight $\lambda^{*}$ can be used to evacuate a biword $W$ from any SSK $\rho^{-1}(T)$ of shape $\delta$, leaving an SSK $\rho^{-1}(U)$ of shape $\gamma$, and such that the lower row of $W$ rectifies to a CT $V$ of shape $\lambda$ such that $T=U \cdot V$.

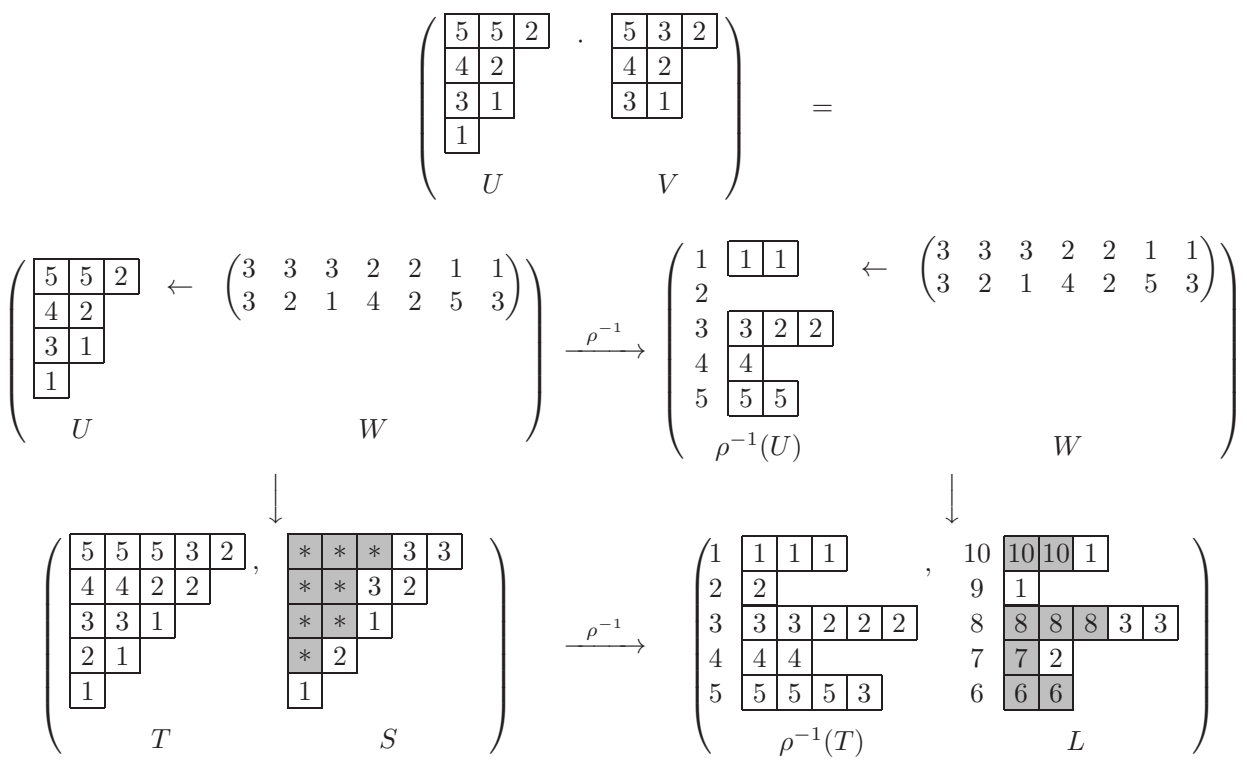

Figure 6. Correspondence example for a term of $\mathcal{A}_{(2,0,3,1,2)} \cdot s_{(3,2,2)}$

In the first direction, suppose we have constructed $L$ from $\rho^{-1}(U) \leftarrow W$ as above. We claim that $L$, including its basement, satisfies the conditions of an SSK. Since we construct $\rho^{-1}(T)$ by adding successive row strips into $\rho^{-1}(U)$, we likewise are constructing $L$ by adding successive row strips to the basement of shape $\gamma$. This implies that the entries within each column of $L$ are distinct. Since we place into $L$ the higher-numbered entries first, this forces the entries within each row of $L$ to be weakly decreasing left to right.

In the construction, suppose that after the addition of some particular cell, row $j$ of the resulting SSK is strictly longer than row $i$ for some $i<j$. As a consequence of the single box case of the Pieri rule, it follows that at every following stage of the construction row $j$ must be strictly longer than row $i$, and so $\delta_{i}<\delta_{j}$. We consider 
triples in $L$.

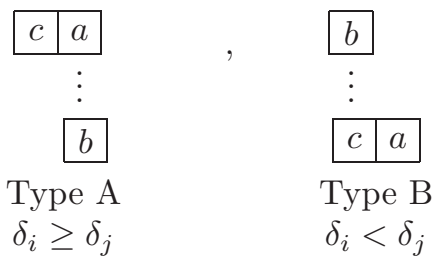

Suppose $L$ has a coinversion type A triple $((i, k),(j, k),(i, k-1))$ with values $(a, b, c)$, as shown. Since rows are weakly decreasing, this would imply $a<b \leq c$, implying that the cell $(i, k)$ is not in the basement and that it was filled after the cell $(j, k)$. But this would imply that just prior to adding cell $(i, k)$, row $j$ was longer than row $i$, which in turn would imply that $\delta_{i}<\delta_{j}$, contradicting the fact that the three cells form a type A triple. Thus $L$ can have no type A coinversion triples.

Suppose $L$ has a coinversion type B triple $((j, k+1),(i, k),(j, k))$ with values $(a, b, c)$, as shown. Since rows are weakly decreasing, this would imply $a \leq b<c$. But that would then imply that cell $(i, k)$ is not in the basement and that it was added before cell $(j, k+1)$, a violation of the Pieri rule. Thus $L$ can have no type B coinversion triples, and so $L$ is a valid SSK.

To see that $\operatorname{colword}(L)$ is a regular contre-lattice word, note that within each column of $L$, the set of values (excluding the basement) in that column is the same as the set of values in the corresponding column of the LR skew CT $S$. We may consider $S$ to be an SSK with basement $b_{i}=2 n-i+1$ of shape $\widetilde{\gamma}$. Since $\operatorname{colword}(S)$ is a regular contre-lattice word, by Proposition 3.1. $S$ is loosely contre-lattice, where the maximum entry in $\operatorname{colword}(S)$ is $\ell(\lambda)$. Since $L$ has the same column sets as $S, L$ is also loosely contre-lattice, and by Proposition $3.1 L$ is contre-lattice, i.e. $\operatorname{colword}(L)$ is a regular contre-lattice word, and so $L$ is an LRS, as claimed.

For the converse direction, assume $L$ is an LRS of shape $\delta / \gamma$ and weight $\lambda^{*}$, and that $\rho^{-1}(T)$ is any SSK of shape $\delta$. We show that we can use $L$ to evacuate a CT from $\rho^{-1}(T)$, as desired. In the process we construct a biword $W$. We know from Proposition 3.2 that the rightmost least entry in $L$ having entry value 1 , call it $x_{1}$, appears at the end of the last row in $L$ of some particular length, which by the Pieri rule implies that we can evacuate the corresponding cell of $\rho^{-1}(T)$, obtaining a value $v$ and leaving a new SSK $\rho^{-1}\left(T^{\prime}\right)$ of the same shape as $L^{\prime}=L-x_{1}$. We record $\left(\begin{array}{l}1 \\ v\end{array}\right)$ as the last biletter of $W$. Since by Proposition 3.3 the remaining SSK $L^{\prime}$ is also contre-lattice, we can repeat the process, constructing the biletters of $W$ in reverse order, until all cells of $L$ and their corresponding cells in $\rho^{-1}(T)$ have been processed, leaving us with a remaining SSK $\rho^{-1}(U)$ of shape $\gamma$ and a biword $W$ whose upper row has weight $\lambda^{*}$. $W$ and $\rho^{-1}(U)$ in turn correspond to a pair of CT $(V, H)$ of the same shape, where clearly $T=U \cdot V$. To see that in fact $H$ is the super CT of shape $\lambda$, consider the parallel step-by-step construction using $S$ to evacuate $T$, where $S$ is the LR skew CT of shape $\widetilde{\delta} / \widetilde{\gamma}$ obtained by sorting the columns of $L$ (including the basement), as illustrated in Figure [6. Evacuating a cell of $\rho^{-1}(T)$ corresponding to a cell $x_{k}$ in $L$ (containing the $k$ th entry of value $x$ in column reading order) corresponds under the bijection $\rho$ to evacuating a cell of $T$ corresponding to the cell $x_{k}$ in $S$, producing the same biword $W$. Since $S$ rectifies to the super CT of shape $\lambda, V$ also has shape $\lambda$. 


\section{Littlewood-RIChardson RUle fOR QUASISymmetric Schur FUnCtions}

Consider an SSK with basement $b_{i}=i$. It is easy to see that if $\gamma_{k}>0$, then in any such SSK $T$ of shape $\gamma$, the cell of $\gamma$ in column one and row $k$ must contain the number $k$. If we consider SSK with an arbitrary increasing basement

$$
1 \leq b_{1}<b_{2}<\cdots<b_{n},
$$

where the $b_{i}$ are not necessarily consecutive, then we can identify $T$ with a unique SSK $\hat{T}$ of shape $\gamma^{+}$obtained by removing the rows of zero length. For such SSK of composition shape, the basement becomes superfluous. This motivates the following definition.

We define a semistandard composition tableau (SSC) of shape $\beta$ (a composition) to be a filling of the diagram $\beta$ which is strictly increasing down the first column, weakly decreasing rightward along each row, and where every triple is an inversion triple. Since by definition the $Q S$ function $\mathcal{S}_{\alpha}$ is the sum of Demazure atoms $\mathcal{A}_{\gamma}$, over all $\gamma$ with $\gamma^{+}=\alpha,(2.2)$ implies that $(\underline{6})$

$$
\mathcal{S}_{\alpha}\left(X_{n}\right)=\sum_{\substack{T \in S S C(n), \\ \text { shape }(T)=\alpha}} \mathbf{x}^{T}
$$

where $\operatorname{SSC}(n)$ is the set of all SSC with entries in $[n]$. These QS functions also satisfy an LR rule. To state it we need to define an analog of LRS. Let $L_{1}$ and $L_{2}$ be elements of $\operatorname{LRS}(n)$, where $L_{1}$ has shape $\delta / \sigma$ and $L_{2}$ has shape $\gamma / \tau$. We declare $L_{1}$ and $L_{2}$ to be equivalent if

(1) $L_{1}$ and $L_{2}$ have the same set of non-basement entries in each column.

(2) $\delta^{+}=\gamma^{+}$, say $\delta^{+}=\gamma^{+}=\beta$.

We define a Littlewood-Richardson skew composition tableau (LRC) to be an equivalence class of $\operatorname{LRS}(n)$, and the collection of such equivalence classes we denote by $\operatorname{LRC}(n)$. Each equivalence class determines a sequence of column sets and a pair of compositions $\beta$ and $\alpha$ which are the underlying compositions of the overall shape and basement shape, respectively, of the elements of the equivalence class. We shall define the shape of the LRC to be this pair of compositions, and by abuse of notation we shall denote the shape by $\beta / \alpha$. (In view of Remark 3.5. the shapes of the respective basements of the elements of a given LRC equivalence class all have the same underlying partition $\alpha$, and hence the shape $\beta / \alpha$ is well-defined.) We can represent an LRC diagrammatically. In Figure 7 we exhibit the four LRC of shape $(4,3,1,2,2) /(3,2,1)$ and content $(1,2,3)$.
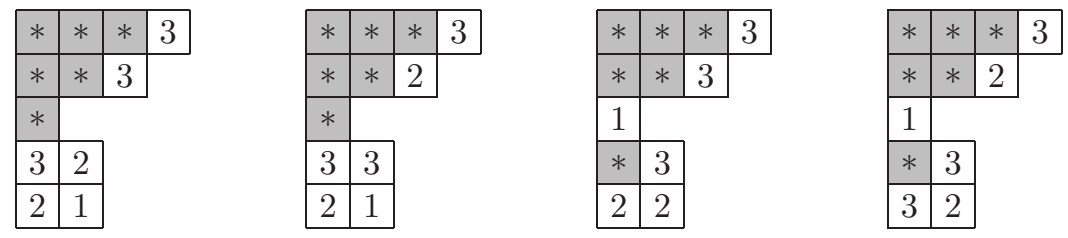

Figure 7 . The four distinct LRC of shape $(4,3,1,2,2) /(3,2,1)$ and content $(1,2,3)$ 
We can now state the LR rule for the product of a QS function and a Schur function.

Theorem 5.1. In the expansion

$$
\mathcal{S}_{\alpha}\left(X_{n}\right) \cdot s_{\lambda}\left(X_{n}\right)=\sum_{\beta} C_{\alpha \lambda}^{\beta} \mathcal{S}_{\beta}\left(X_{n}\right),
$$

the coefficient $C_{\alpha \lambda}^{\beta}$ is the number of elements in $\operatorname{LRC}(n)$ of shape $\beta / \alpha$ with content $\lambda^{*}$.

Proof. We make use of (5.1) and (2.1). The SSC are trivially in bijection with the SSKI, that is, the SSK with $b_{i}=i$. Hence the mapping $\rho: \operatorname{SSKI}(n) \rightarrow \operatorname{CT}(n)$ can be viewed as a bijection between SSC $D$ and CT $\rho(D)$ whose columns are just the respective sorted column sets of $D$. In view of the proof of Theorem 4.1, it suffices to provide a bijection $(U, V) \leftrightarrow(T, S)$ between pairs $(U, V)$ of $\mathrm{CT}$, where $\rho^{-1}(U)$ is an SSC of shape $\alpha$ and $V$ has shape $\lambda$, and pairs $(T, S)$, where $T$ is the CT $T=U \cdot V$, with $\rho^{-1}(T)$ an SSC of shape $\beta$, and $S$ is an LRC of weight $\lambda^{*}$ and shape $\beta / \alpha$.

We make use of the bijection $(U, V) \leftrightarrow(T, L)$ as constructed in the proof of Theorem 4.1. Suppose here, as in the proof, that $(U, V)$ is a pair of CT with entries in $[n]$. Under the bijection $\rho^{-1}: \mathrm{CT}(n) \rightarrow \operatorname{SSKI}(n)$ we can map $U$ and $T=U \cdot V$, respectively, to SSKI with $n$ rows, say $\rho^{-1}(U)$ of shape $\gamma$ and $\rho^{-1}(T)$ of shape $\delta$. Under the bijection from the proof of Theorem 4.1 $\rho^{-1}(T)$ is paired with an LRS $L$ of weight $\lambda^{*}$ and shape $\delta / \gamma$. Thus the pair $(U, V)$ determines a unique pair $(T, S)$, where $S$ is the LRC of shape $\delta^{+} / \gamma^{+}$that is the equivalence class of $L$.

Conversely, suppose we have a pair $(T, S)$ where $\rho^{-1}(T)$, viewed as an SSC, has shape $\beta$ and $S$ is an element of $\operatorname{LRC}(n)$ of weight $\lambda^{*}$ and of shape $\beta / \alpha$. Then under the bijection $\rho^{-1}: \operatorname{CT}(n) \rightarrow \operatorname{SSKI}(n), \rho^{-1}(T)$ is an SSKI of shape $\delta$, where $\ell(\delta)=n$ and $\delta^{+}=\beta$. Now by Proposition 3.4 the existence of $S$ implies that there is a unique LRS $L$ of shape $\delta / \gamma$ for some $\gamma$ with $\widetilde{\gamma}=\widetilde{\alpha}$ and having the same column sets of entries as the elements of $S$. As mentioned in Remark 3.5, the construction in the proof of Proposition 3.4 implies that $\gamma^{+}=\alpha$, that is, $L$ is in fact an element of $S$. Thus the pair $(T, S)$ determines a unique pair $(T, L)$. Furthermore, $L$ has weight $\lambda^{*}$. Under the bijection from the proof of Theorem 4.1 $(T, L)$ is paired with a pair of $\mathrm{CT}(U, V)$ where $T=U \cdot V, V$ has shape $\lambda$, and $\rho^{-1}(U)$ has shape $\gamma$, which implies that $\rho^{-1}(U)$, when viewed as an SSC, has shape $\gamma^{+}=\alpha$, as desired.

\section{Littlewood-Richardson RUle For DemaZure Characters}

A Littlewood-Richardson skew key (LRK) of shape $\delta / \gamma$ is an SSK of shape $\delta / \gamma$ with basement $b_{i}=n+i$, where $n=\ell(\delta)=\ell(\gamma)$ and whose column reading word is a regular contre-lattice word. We let $\operatorname{LRK}(n)$ denote the set of LRK with entries in $[n]$. Figure 8 provides an example of an LRK of shape $(5,1,3,2,4) /(2,0,1,2,3)$ and colword $=3323121$.

We can now state our LR rule for the product of a Schur function and a Demazure character.

Theorem 6.1. In the expansion

$$
\kappa_{\gamma}\left(X_{n}\right) \cdot s_{\lambda}\left(X_{n}\right)=\sum_{\delta} b_{\gamma \lambda}^{\delta} \kappa_{\delta}\left(X_{n}\right)
$$




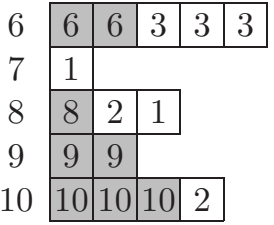

FIGURE 8. An LRK with $n=5$ and column reading word 3323121

the coefficient $b_{\gamma \lambda}^{\delta}$ is the number of elements in LRK $(n)$ of shape $\delta^{*} / \gamma^{*}$ with content $\lambda^{*}$.

Proof of Theorem [6.1. Recall [11, [16] that the Demazure characters can be obtained from the Demazure atoms:

$$
\kappa_{\gamma}=\sum_{\beta \geq \gamma^{*}} \mathcal{A}_{\beta},
$$

where the sum is over all compositions $\beta$ which are weakly above $\gamma^{*}$ in the Bruhat order. (Given a weak composition $\gamma$, let $\pi(\gamma)$ be the permutation of minimal length which arranges the parts of $\gamma$ into non-increasing order. Then we define $\beta \geq \alpha$ if and only if $\pi(\beta) \leq \pi(\alpha)$ in the usual (strong) Bruhat order on permutations.) We substitute the formula (4.1) for the multiplication of a Demazure atom and a Schur function to obtain the following formula for the left hand side of (6.1):

$$
\kappa_{\gamma} \cdot s_{\lambda}=\sum_{\beta \geq \gamma^{*}} \mathcal{A}_{\beta} \cdot s_{\lambda}=\sum_{\beta \geq \gamma^{*}} \sum_{\delta} a_{\beta \lambda}^{\delta} \mathcal{A}_{\delta},
$$

where $a_{\beta \lambda}^{\delta}$ is the number of elements of $\operatorname{LRS}(n)$ of shape $\delta / \beta$ with content $\lambda^{*}$. To prove that

$$
\sum_{\alpha} b_{\gamma \lambda}^{\alpha} \kappa_{\alpha}=\sum_{\beta \geq \gamma^{*}} \sum_{\delta} a_{\beta \lambda}^{\delta} \mathcal{A}_{\delta},
$$

we further expand the left hand side of (6.3) to see that our theorem is equivalent to the identity

$$
\sum_{\alpha} b_{\gamma \lambda}^{\alpha} \sum_{\delta \geq \alpha^{*}} \mathcal{A}_{\delta}=\sum_{\delta} \sum_{\beta \geq \gamma^{*}} a_{\beta \lambda}^{\delta} \mathcal{A}_{\delta} .
$$

Each coefficient $b_{\gamma \lambda}^{\alpha}$ appearing on the left hand side of (6.4) is the coefficient of every Demazure atom $\mathcal{A}_{\delta}$ such that $\delta \geq \alpha^{*}$. Since the Demazure atoms are linearly independent, comparing the coefficients of $\mathcal{A}_{\delta}$ on both sides of (6.4) reduces our identity to

$$
\sum_{\delta \geq \alpha^{*} \supseteq \gamma^{*}} b_{\gamma \lambda}^{\alpha}=\sum_{\delta \supseteq \beta \geq \gamma^{*}} a_{\beta \lambda}^{\delta}
$$

for fixed $\delta$ and $\gamma$. It therefore suffices to fix $\delta$ and $\gamma$ and to find a bijection between the set $K$ of all LRK of shape $\alpha^{*} / \gamma^{*}$ with content $\lambda^{*}$ where $\alpha^{*} \leq \delta$ in Bruhat order and the set $L$ of all LRS of shape $\delta / \beta$ with content $\lambda^{*}$ where $\beta \geq \gamma^{*}$ in Bruhat order.

We begin with the forward direction of the map $\phi: K \mapsto L$. Let $K$ be an LRK with content $\lambda^{*}$ and shape $\alpha^{*} / \gamma^{*}$, and assume $\alpha^{*} \leq \delta$ in Bruhat order. By 
Proposition 3.4 there exists a unique LRS $L$ of shape $\delta / \beta$ for some $\beta$ a permutation of $\gamma$ and having the same column sets as $K$. Map the LRK $K$ to this LRS $L$. To show that the map takes $K$ into the appropriate set, we must prove that $\beta \geq \gamma^{*}$ in Bruhat order.

To see this, let $\gamma_{K}$ be the overall shape of $K$ and $\gamma_{L}$ the overall shape of $L$, and then apply the following iterative argument. The overall shape of $L$ is weakly higher than the reverse of the shape of $K$ by construction, so $\gamma_{K}{ }^{*} \leq \gamma_{L}$. In the construction given by Proposition 3.4. consider the first entry in $K$ that is mapped to $L$. This entry is mapped to a row $\left(r_{1}\right)_{L}$ of $L$ weakly higher than the reverse of the row $\left(r_{1}\right)_{K}$ of $K$ from which it is removed, since the largest part of $\gamma_{L}$ appears before the largest part of $\gamma_{K}{ }^{*}$. Subtract one from the $\left(r_{1}\right)_{L}$ part of $\gamma_{L}$ and the $\left(r_{1}\right)_{K}$ part of $\gamma_{K}{ }^{*}$ to obtain new compositions $\gamma_{K}{ }^{*} \leq \gamma_{L}$. Repeat this procedure until there are no remaining non-basement entries in $K$. The resulting compositions $\gamma_{K}{ }^{*}$ and $\gamma_{L}$ are the shapes of the respective basements and satisfy $\gamma_{K}{ }^{*} \leq \gamma_{L}$. Therefore the basement of $L$ is indeed higher in Bruhat order than the reverse basement of $K$. (See Figure 9 for an example.)

We have now shown that $L$ is an LRS in the desired set. Proposition 3.4 shows that $L$ is unique, therefore the map $\phi$ is injective. We describe the inverse of the map $\phi$ to prove that the map is surjective. Consider an arbitrary LRS $L$ of shape $\delta / \beta$ and content $\lambda^{*}$, and let $\gamma$ be a rearrangement of $\beta$ such that $\gamma^{*} \leq \beta$ in Bruhat order. We need to map $L$ back to an LRK of shape $\alpha^{*} / \gamma^{*}$ for some $\alpha^{*} \leq \delta$ in Bruhat order and having the same column sets as $L$. (Note that all LRK in the pre-image have the same fixed basement of shape $\gamma^{*}$.) Let $K^{0}$ be the basement diagram of type $b_{i}=n+i$ and of shape $\gamma^{*}$. This is the basement on which the LRK will be built. Begin with the leftmost column of the LRS $L$ and the largest non-basement entry in this column. Place this entry in the highest available row of this column in $K^{0}$, i.e. in an empty cell not part of the basement such that the entry to its left is non-empty and greater than or equal to our insertion entry. Call the resulting SSK $K^{1}$. Repeat with the second largest entry in the leftmost column of $L$ to create $K^{2}$. Continue this procedure until all of the non-basement entries in the leftmost column of $L$ have been placed into the skyline diagram. Repeat with each column of $L$ from left to right until all of the non-basement entries of $L$ have been inserted into the SSK $K$. We must prove that $K$ is indeed an LRK, say of shape $\alpha^{*} / \gamma^{*}$, and that $\alpha^{*} \leq \delta$ in Bruhat order.

The rows of $K$ are weakly decreasing by construction, so we must check that the triple conditions are satisfied. We consider triples in $K$.

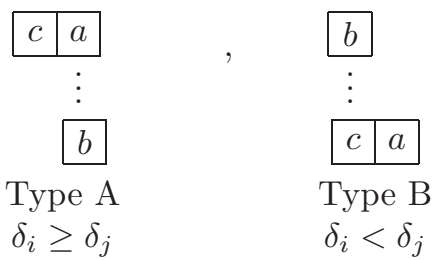

Suppose $K$ has a coinversion type A triple $((i, k),(j, k),(i, k-1))$ with values $(a, b, c)$ as shown. Since the rows of $K$ are weakly decreasing, this would imply that $a<b \leq c$ and therefore that the cell $(j, k)$ is not in the basement and was filled before the cell $(i, k)$. But since the cell $(i, k-1)$ was filled before $(i, k)$ was filled, the entry $b$ would have been inserted into the cell $(i, k)$, a contradiction. Thus $K$ can have no type A coinversion triples. 


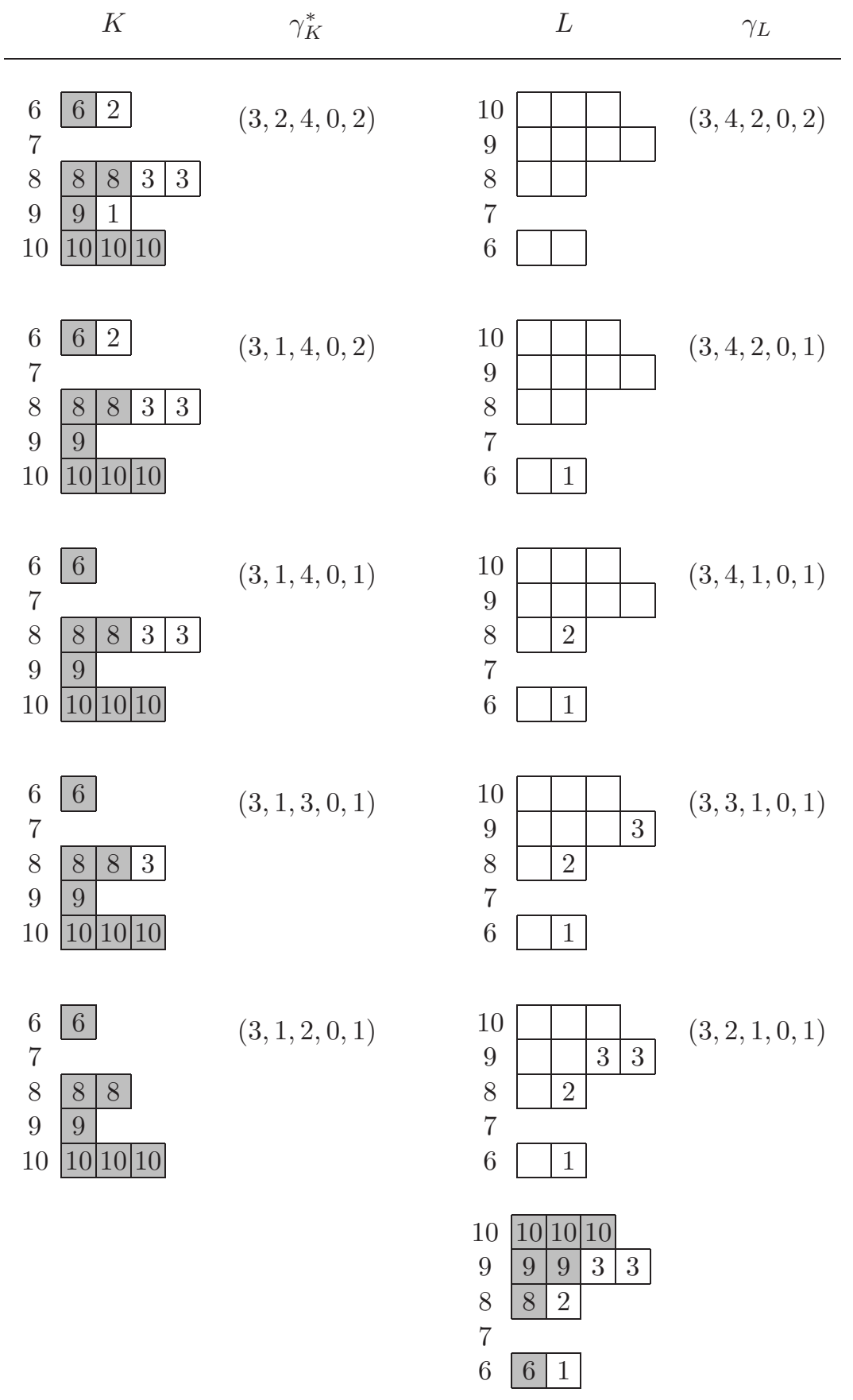

Figure 9. Constructive comparison of the basements of $K$ and $L$ 
Suppose $K$ has a coinversion type $B$ triple $((j, k+1),(i, k),(j, k))$ with values $(a, b, c)$, as shown. Since the rows of $K$ are weakly decreasing, this would imply $a \leq b<c$. That would then imply that the cell $(j, k+1)$ is not in the basement, and was added after the cell $(i, k+1)$, for otherwise the entry $a$ would be inserted into the cell $(i, k+1)$. Therefore the entry in cell $(i, k+1)$ is greater than the entry in $(j, k+1)$ and will be filled first. Continuing in this manner implies that $\delta_{i} \geq \delta_{j}$, contradicting the assumption that the three cells form a type B triple. Thus $K$ can have no type B coinversion triples.

We invoke Proposition 3.1 to see that the diagram $K$ is contre-lattice, since $L$ is contre-lattice and the map from $L$ to $K$ preserves the column sets of the diagrams. We claim furthermore that $\alpha^{*} \leq \delta$ in Bruhat order. To begin with, we have by assumption $\gamma^{*} \leq \beta$, where $\gamma^{*}$ is the shape of $K^{0}$, the basement of $K$, and $\beta$ is the shape of the basement of $L$. As the first non-basement entry of $L$ is mapped to produce $K^{1}$, it will appear in a weakly higher row of $K^{1}$ than its appearance in $L$ by construction. The resulting shape of $K^{1}$ will therefore remain weakly lower in Bruhat order than the union of the basement of $L$ and this entry. Iterating this argument implies that the overall shape $\alpha^{*}$ of $K$ is weakly lower in Bruhat order than the overall shape $\delta$ of $L$.

6.1. Recovering the classical Littlewood-Richardson rule. Every Schur function is a Demazure character; in particular $s_{\mu}\left(X_{n}\right)=\kappa_{\mu^{*}}\left(X_{n}\right)$. Theorem 6.1 is therefore a generalization of the classical Littlewood-Richardson rule. Consider the product

$$
\begin{aligned}
s_{\mu}\left(X_{n}\right) \cdot s_{\lambda}\left(X_{n}\right) & =\kappa_{\mu^{*}}\left(X_{n}\right) \cdot s_{\lambda}\left(X_{n}\right) \\
& =\sum_{\delta} b_{\mu^{*} \lambda}^{\delta} \kappa_{\delta}\left(X_{n}\right) .
\end{aligned}
$$

We claim that $\sum_{\delta} b_{\mu^{*} \lambda}^{\delta} \kappa_{\delta}\left(X_{n}\right)=\sum_{\nu} c_{\mu \lambda}^{\nu} s_{\nu}\left(X_{n}\right)$, where $c_{\mu \lambda}^{\nu}$ is the number of LR skew CT with shape $\nu / \mu$ and content $\lambda^{*}$. To see this, let $L$ be an arbitrary element in $\operatorname{LRK}(n)$ of shape $\delta^{*} / \mu$ and content $\lambda^{*}$. The basement of $L$ is the partition $\mu$. If the shape of $\delta^{*}$ is not a partition, then consider two rows $i$ and $j$ of $\delta^{*}$ such that $i<j$ but row $j$ is strictly longer than row $i$. Let $C$ be the column containing the rightmost entry of the basement in row $j$. This entry, together with the entry immediately to its right and the entry in row $i$ of column $C$, form a type $B$ coinversion triple. Therefore the shape $\delta^{*}$ must be a partition, and so $\kappa_{\delta}\left(X_{n}\right)$ is the Schur function $s_{\delta^{*}}\left(X_{n}\right)$.

We already know that the row entries of $L$ weakly decrease left to right, and, since $\delta^{*}$ and $\mu$ are partitions, all inversion triples must be of type $A$. Consequently our non-basement column entries decrease top to bottom. Thus, $L$ is a skew CT. Lastly, note that colword $(L)$ is regular contre-lattice if and only if $L$ is furthermore an LR skew CT by Proposition 2.3. Therefore Theorem 6.1 reduces to the classical Littlewood-Richardson rule whenever $\kappa_{\gamma}\left(X_{n}\right)$ is a Schur function.

\section{ACKNOWLEDGMENTS}

The first author was supported in part by NSF grant DMS-0553619 and DMS0901467. The third author was supported in part by NSF postdoctoral research 
fellowship DMS-0603351. The second and fourth authors were supported in part by the National Sciences and Engineering Research Council of Canada. The authors would like to thank the Banff International Research Station and the Centre de Recherches Mathématiques, where some of the research took place. The authors would also like to thank the referee for insightful comments and suggestions.

\section{REFERENCES}

1. Akin, K., Buchsbaum, D., Weyman, J., Schur functors and Schur complexes, Adv. Math., 44 (1982), 207-278. MR658729 (84c:20021)

2. Demazure, M., Désingularisation des variétés de Schubert, Ann. E. N. S., 6 (1974), 163-172.

3. Fulton, W., Young Tableaux, Cambridge University Press, Cambridge, UK, 1997. MR 1464693 (99f:05119)

4. Gessel, I., Multipartite $P$-partitions and inner products of skew Schur functions, Contemp. Math., 34 (1984), 289-317. MR777705 (86k:05007)

5. Haglund, J., Haiman, M., Loehr, N., A combinatorial formula for nonsymmetric Macdonald polynomials, Amer. J. of Math., 103 (2008), 359-383. MR2405160 (2009f:05262)

6. Haglund, J., Luoto, K., Mason, S., van Willigenburg, S., Quasisymmetric Schur functions, J. Combin. Theory Ser. A, in press. Also available at arXiv:0810.2489.

7. van der Kallen, W., Lectures on Frobenius splittings and B-modules, Springer, New York, USA, 1993.

8. Kogan, M., RC-graphs and a generalized Littlewood-Richardson rule, Int. Math. Res. Not., 15 (2001), 765-782. MR.1849481 (2002m:05197)

9. Kohnert, A., Multiplication of a Schubert Polynomial by a Schur Polynomial, Ann. Comb., 1 (1997), 367-375. MR.1630751 (99c:05195)

10. Lakshmibai, V., Magyar, P., Standard monomial theory for Bott-Samelson varieties, C. R.Acad. Sci. Paris Ser. I Math., 324 (1997), 1211-1215. MR1456289 (98g:14065)

11. Lascoux, A., Schützenberger, M.-P., Keys and standard bases, Invariant Theory and Tableaux, IMA Volumes in Math and its Applications (D. Stanton, Ed.), Southend on Sea, UK, 19 (1990), 125-144. MR.1035493 (91c:05198)

12. Macdonald, I., A new class of symmetric polynomials, Sém. Lothar. Combin., $\mathbf{3 7 2}$ (1988).

13. Macdonald, I., Symmetric functions and Hall polynomials, 2nd Edition, Oxford University Press, New York, USA, 1995. MR1354144 (96h:05207)

14. Macdonald, I., Affine Hecke algebras and orthogonal polynomials, Astérisque, 237 (1996), 189-207. MR1423624 (99f:33024)

15. Mason, S., A decomposition of Schur functions and an analogue of the Robinson-SchenstedKnuth algorithm, Sém. Lothar. Combin., 57 (2008), B57e.

16. Mason, S., An explicit construction of type A Demazure atoms, J. Algebraic Combin., 29 (2009), 295-313. MR2496309

17. Polo, P., Variétés de Schubert et excellentes filtrations, Astérisque, 173-174 (1989), 281-311. MR:1021515 (91b:20056)

18. Reiner, V., Shimozono, M., Key polynomials and a flagged Littlewood-Richardson rule, J. Combin. Theory Ser. A, 70 (1995), 107-143. MR1324004(96i:05175)

19. Reiner, V., Shimozono, M., Flagged Weyl modules for two-column shapes, J. Pure Appl. Algebra, 141 (1999). MR.1705973 (2000f:05088)

20. Reiner, V., Shimozono, M., Straightening for standard monomials on Schubert varieties, J. Algebra, 195 (1997), 130-140. MR1468886 (98h:14063)

21. Reiner, V., Shimozono, M., Percentage-avoiding, northwest shapes and peelable tableaux, J. Combin. Theory Ser. A, 82 (1998), 1-73. MR1616579 (2000a:05220)

22. Stanley, R., Enumerative Combinatorics, Volume 2, Cambridge University Press, Cambridge, UK, 1999. MR 1676282 (2000k:05026)

23. Taylor, B., A straightening algorithm for row-convex tableaux, J. Algebra, 236 (2001), 155191. MR.1808350(2002a:20047) 
Department of Mathematics, University of Pennsylvania, Philadelphia, Pennsylvania 19104-6395

E-mail address: jhaglund@math.upenn.edu

Department of Mathematics, University of British Columbia, Vancouver, British Columbia, Canada V6T $1 \mathrm{Z} 2$

E-mail address: kwluoto@math.ubc.ca

Department of Mathematics, University of California at San Diego, San Diego, CalIFORNIA 92093

E-mail address: skmason@math.ucsd.edu

Current address: Department of Mathematics, Wake Forest University, Winston-Salem, North Carolina 27109

E-mail address: masonsk@wfu.edu

Department of Mathematics, University of British Columbia, Vancouver, British Columbia, Canada V6T $1 \mathrm{Z2}$

E-mail address: steph@math.ubc.ca 